\title{
On the minimum volume simplex enclosure problem for estimating a linear mixing model
}

\author{
E. M. T. Hendrix · I. García · J. Plaza · A. Plaza
}

Received: 19 January 2012 / Accepted: 11 February 2012 / Published online: 26 February 2012

(C) The Author(s) 2012. This article is published with open access at Springerlink.com

\begin{abstract}
We describe the minimum volume simplex enclosure problem (MVSEP), which is known to be a global optimization problem, and further investigate its multimodality. The problem is a basis for several (unmixing) methods that estimate so-called endmembers and fractional values in a linear mixing model. We describe one of the estimation methods based on MVSEP. We show numerically that using nonlinear optimization local search leads to the estimation results aimed at. This is done using examples, designing instances and comparing the outcomes with a maximum volume enclosing simplex approach which is used frequently in unmixing data.
\end{abstract}

Keywords Application - Spectral unmixing · Endmembers · Principal components · Minimum volume $\cdot$ Maximum volume $\cdot$ Estimation

This work has been supported by the European Community Marie Curie Research Training Networks Program (MRTN-CT-2006-035927), Hyperspectral Imaging Network (HYPER-I-NET), the Spanish Ministry of Science and Innovation (TIN2008-01117 and AYA2008-05965-C04-02), Junta de Andalucía (P11-TIC-7176) and Junta de Extremadura (PRI09A110) in part financed by the European Regional Development Fund (ERDF). Eligius Hendrix is a fellow of the Spanish "Ramon y Cajal" program cofinanced by the European Social Fund.

E. M. T. Hendrix $(\bowtie)$. I. García

Computer Architecture Department, Universidad de Málaga, Málaga, Spain

e-mail: eligius@uma.es

I. García

e-mail: igarciaf@uma.es

E. M. T. Hendrix

Operations Research and Logistics, Wageningen University, Wageningen, The Netherlands

J. Plaza · A. Plaza

Department of Technology of Computers and Communications, Universidad de Extremadura, Cáceres, Spain e-mail:jplaza@unex.es

A. Plaza

e-mail: aplaza@unex.es 


\section{Introduction}

The problem to enclose a number of points by a body of certain shape is often a global optimization problem. The minimum volume simplex enclosure problem (MVSEP) minimizes the volume of a simplex that encloses a given set of points. Initially, [3] showed that the solution of MVSEP can be used for so-called unmixing of sensor data; having (high dimensional) data from multispectral imaging sensors one tries to unfold (unmix) them into components. The underlying statistical model under consideration is the so-called linear mixing model:

$$
y=X a+\varepsilon
$$

where $y$ is an $m$-vector of observed properties, $X$ is an $m \times(n+1)$ matrix with the properties of pure constituents, also called endmembers, and $a$ is an $(n+1)$-vector of fractions, also called abundances. Often $\varepsilon$ is taken as a noise $m$-vector with components that are i.i.d. Gaussian random variables with a standard deviation of $\sigma, \varepsilon \sim N\left(0, \sigma^{2} E\right), E$ the identity matrix.

Given a set of observations $y_{1}, \ldots, y_{r}$, the estimation problem is to recover $X$ and to obtain for each observation $y_{k}$ its fractional composition $a_{k}$ that should be nonnegative and its elements summing up to one, i.e. it lays on the unit simplex. The model has important applications in geology ([8]) where soil samples are analyzed and decomposed in its basic constituents and in the analysis of remotely sensed hyperspectral data, where the estimation is called endmember extraction and spectral unmixing. An overview is given by [6] and in [2] an extensive description of underlying concepts and developed methods is provided. In this field, besides the use of an MVSEP approach, also a maximum volume "inscribed" simplex concept exists and is the base of the so-called N- FINDR algorithm extensively described in [10].

Section 2 discusses the MVSEP and illustrates its multimodal character. It is put besides the maximum volume "inscribed" simplex problem. In Sect. 3, a procedure is described for estimating parameter values of the linear mixing model based on MVSEP. Section 4 investigates the relation between input noise and the variability of estimates with numerical instances, when local searches are used to generate solutions of MVSEP. Finally, conclusions are drawn in Sect. 5.

\section{Minimum volume and maximum volume simplex problems}

We first focus on the MVSEP and then show its relation with the maximum volume problem. The problem of finding the minimum volume enclosing simplex of a set of points $z_{k} \in \mathbb{R}^{n}, \forall k=1, \ldots, r$ consists of finding a set of $n+1$ affine independent vertices $v_{i}$ such that $V=\left[v_{1}, \ldots, v_{n+1}\right]$ is the minimum of

$$
\min _{\hat{V}}\left\{f(\hat{V}):=\left|\operatorname{det}\left(\begin{array}{c}
\hat{V} \\
\mathbf{1}^{T}
\end{array}\right)\right|\right\} \text { s.t.: } \beta_{k}=\left(\begin{array}{c}
\hat{V} \\
\mathbf{1}^{T}
\end{array}\right)^{-1}\left(\begin{array}{c}
z_{k} \\
1
\end{array}\right) \geq 0, \forall k
$$

where 1 is the all-ones vector. MVSEP (2) is a nonlinear optimization problem with $n \times(n+1)$ variables and $r \times(n+1)$ constraints. Note that vector $\beta_{k}$ of point $k$ denotes how $z_{k}$ can be written as a convex combination of the columns of $V$. If it has components that are zero, $z_{k}$ is on the boundary of the simplex $\hat{S}=\operatorname{con} v(\hat{V})$. Corresponding to the terminology of active (binding) constraints, we call the points at the boundary active points. 


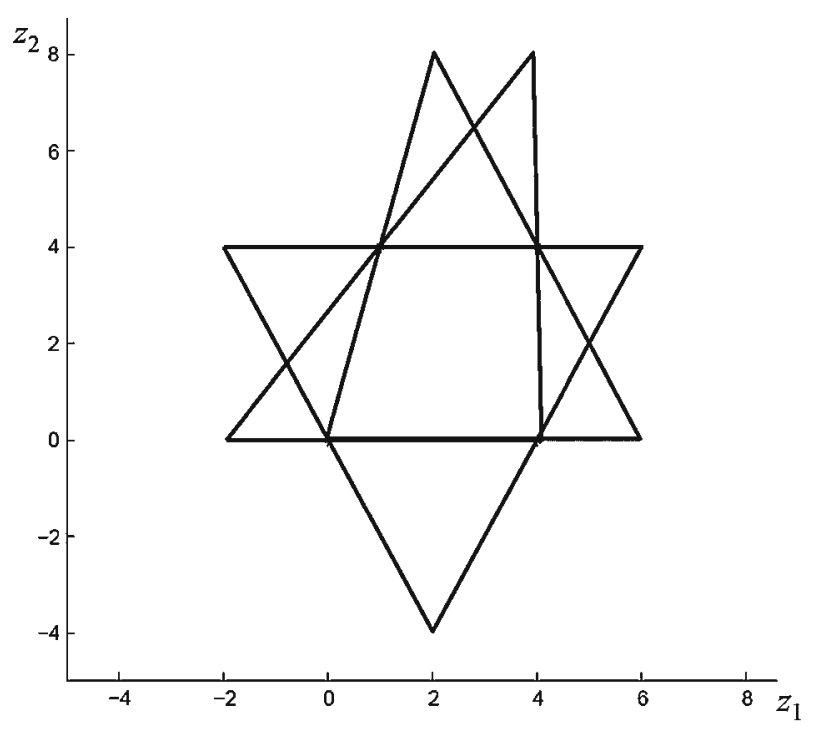

Fig. 13 enclosing simplices of 4 points

Enclosing a set of points with a minimum volume shape has specific mathematical characteristics. For any convex shape where one minimizes the volume, the points in the interior of the convex hull do not have to be taken into account. However, the determination of the points that are on the boundary of the convex hull is of the same complexity as finding the minimum volume shape [7]. Usually, a limited number of active points can be found on the boundary of the minimum volume shape. In [4], several examples for enclosing with spheres (the Chebychev problem) and with hyper-rectangles are given. Enclosing a set of points by a minimum radius sphere is a convex problem and therefore relatively easy to solve. Khachiyan and Todd [7], Sun and Freund [9] discuss the complexity to determine for a set of points the enclosing and enclosed ellipsoids. In [4] a small instance with only 4 points is used to illustrate the complexity of the enclosing hyperrectangle problem. We generate a 4-point problem for (2) as an example.

Example 1 Given matrix $Z=\left[z_{1}, \ldots, z_{4}\right]$ with data $Z=\left(\begin{array}{llll}0 & 4 & 4 & 1 \\ 0 & 0 & 4 & 4\end{array}\right)$. Problem (2) has several local minima for this instance. They can be found by running nonlinear optimization algorithms from several starting points. In this example, the global minimum solution in terms of the enclosing simplex is not unique. In Fig. 1, three enclosing simplices are given. Vertex matrices $\left(\begin{array}{lll}0 & 6 & 2 \\ 0 & 0 & 8\end{array}\right),\left(\begin{array}{rrr}-2 & 4 & 4 \\ 0 & 0 & 8\end{array}\right)$ give volume 48 and $\left(\begin{array}{rrr}-2 & 6 & 2 \\ 4 & 4 & -4\end{array}\right)$ represents a simplex of volume 64 . The latter is called a local minimum solution; moving vertices a bit such that it still encloses the points increases its volume. Moreover, any convex combination of the matrices with volume 48 also has a volume of 48 . This means that the number of global minimum solutions (simplices) of (2) in this example is infinite.

First of all one should keep in mind that, in general, having a global optimum enclosing simplex implies $(n+1)$ ! solution matrices $V$, as any permutation of the columns in $V$ represents the same simplex. When using (2) to estimate a constituent matrix, one has to allocate vertices $v_{i}$ to constituents during an interpretation of estimation results. Besides symmetry, 


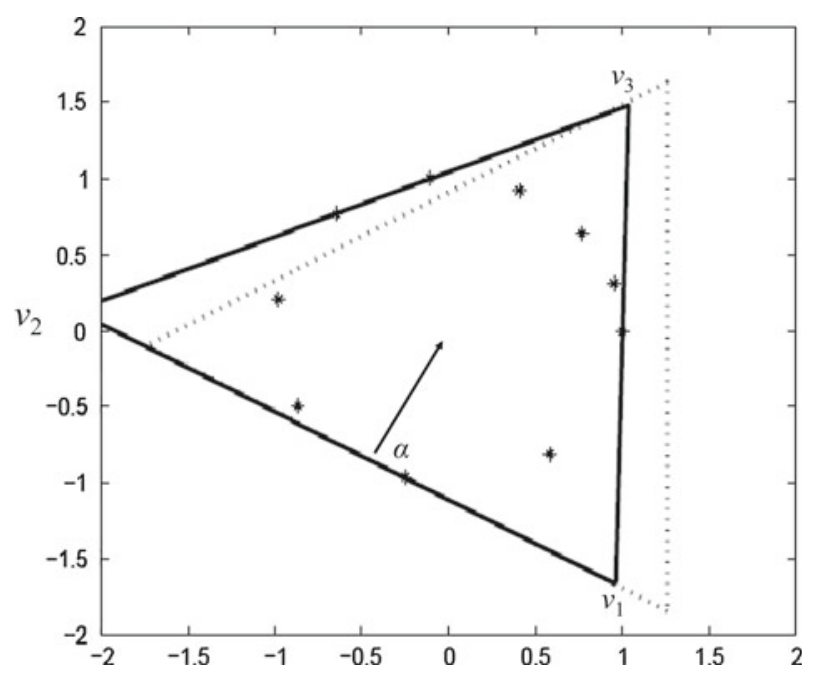

Fig. 2 Enclosing 10 points on the unit ball. Dotted simplex is first guess as starting simplex of a local search algorithm with fixed line simplex as result

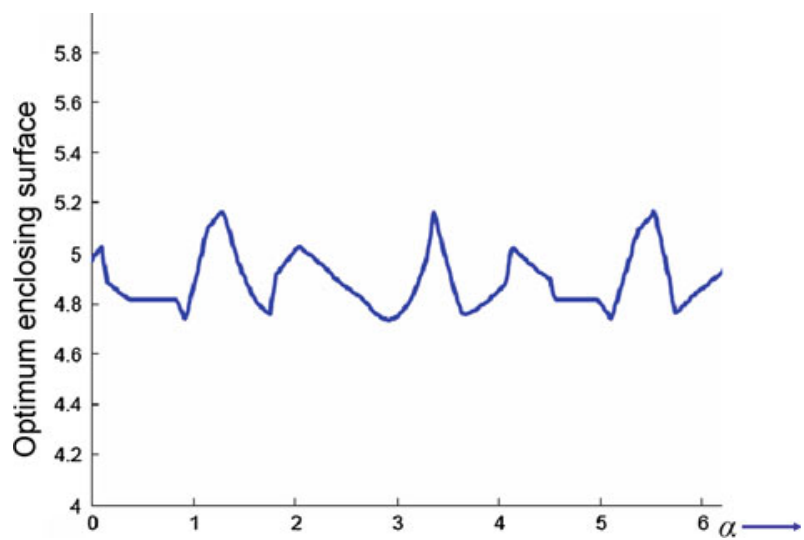

Fig. 3 Minimum volume of enclosing simplex when fixing angle $\alpha$ of first edge

MVSEP (2) is harder for an instance $Z$ representing a "round" cloud of points. To illustrate this, we construct an instance where the points are distributed over the unit ball as our next example.

Example 2 To give a good picture of multimodality is not straightforward; even in the 2-dimensional cases the MVSEP has 6 parameters to optimize. Therefore we construct a parameterized example where the angle of the first edge $\alpha$ of the simplex is fixed and we optimize over the other parameters as illustrated in Fig. 2.

The basis of the case is enclosing the unit ball (any ellipse also would do) with a (regular) simplex of volume $3 \sqrt{3} \approx 5.2$. For the illustration, we generate 10 points over the unit ball and solve MVSEP by fixing $\alpha$ and the corresponding edge and minimizing over the rest of the parameters. The result of this exercise has been drawn in Fig. 3. It shows the minimum volume for varying value of $\alpha$. The minima in Fig. 3 are local minima of problem (2). 

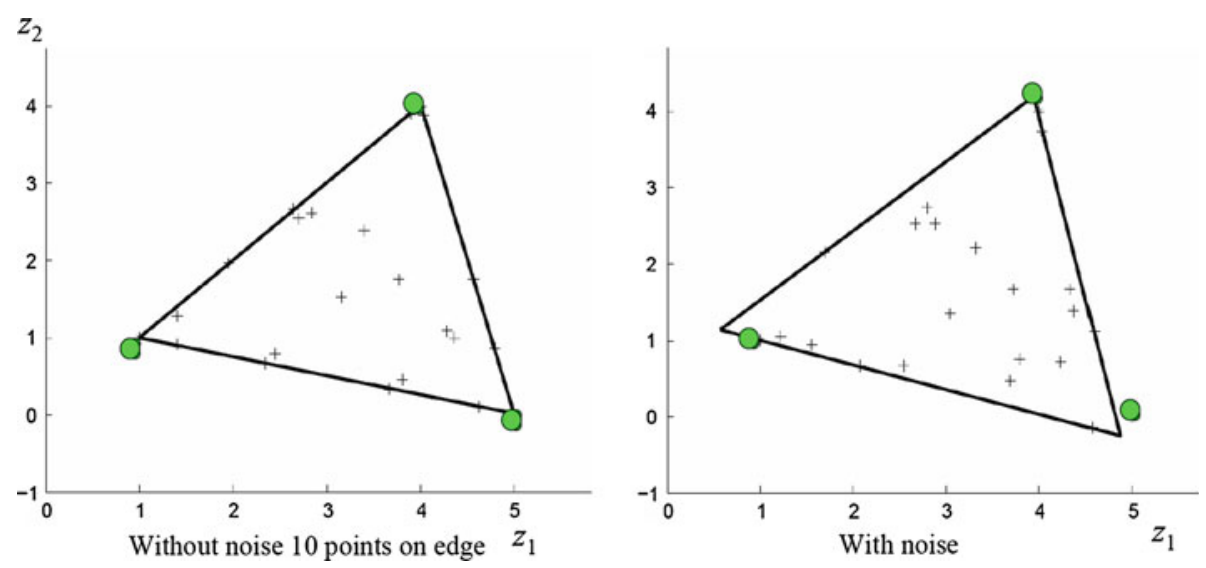

Fig. 43 Endmembers, 20 observations in 2D with enclosing simplex $\hat{S}$

The instance shows that the number of optima, when points $z_{k}$ are 2-dimensional, increases with the number $r$ of points as long as they are in the convex hull. This is confirmed by the findings of [11], which constructs a specific algorithm for 3-dimensional instances.

Now consider the linear mixing model, where MVSEP is applied in estimation methods. Let the points $z_{k}$ be observations of model

$$
z=V a+\xi
$$

where $\xi$ is an $n$-vector of Gaussian i.i.d. variates. $V$ is now a $n \times(n+1)$ matrix of endmembers. So, (3) is a special case of model (1) with $n=m$. Notice that in practice $m>>n$, i.e. the number of measured properties of a sample is much higher than the number of constituents. The question here is: if $z_{k}$ are samples from a lower dimensional model (3), does a permutation of the columns of a solution $\hat{V}=\left[\hat{v}_{1}, \ldots, \hat{v}_{n+1}\right]$ represent an approximation of the endmembers in $V$ ? In other words, how well does simplex $\hat{S}=\operatorname{con} v(\hat{V})$ approximate $S=\operatorname{con} v(V)$ and how multimodal is (2)?

Example 3 Consider matrix of endmembers $V=\left(\begin{array}{lll}1 & 4 & 5 \\ 1 & 4 & 0\end{array}\right)$ depicted in Fig. 4 by dots. In total, 20 points are generated as convex combinations of the columns of $V$ and depicted as crosses in the leftmost picture of Fig. 4 where 10 are a combination of 2 endmembers and 10 are a combination of 3 endmembers. Only if the number of points on the boundary is very low, enclosing simplex $\hat{S}$ does not reflect matrix $V$ as vertices. Simplex $S$ has a volume of 15 . We add noise with $\sigma=0.1$ to simulate observations of (3) and obtain the points in the right picture of Fig. 4 . They are enclosed by a simplex $\hat{S}$ of volume 17 . Notice that less points are now located at the boundary. It may be intuitively clear that there should be at least 4 active points to obtain the minimum volume simplex in $2 \mathrm{D}$.

Example 3 shows several features of using the MVSEP for estimating endmembers. First of all, if a sufficient number of points are at the boundary (their fraction vector $a$ contains zeros) and there is no noise, $\hat{S}=S$, i.e. we recover the endmembers and there is one (local) optimal enclosing simplex; it is sufficient to apply nonlinear optimization local search.

Secondly, if points are well spread over the boundary, the cloud of points is not "round" and consequently problem (2) has either one global minimum simplex, or local nonglobal minima that are close to the global one, as well in function value as in (permutation of) matrix $\hat{V}$. 


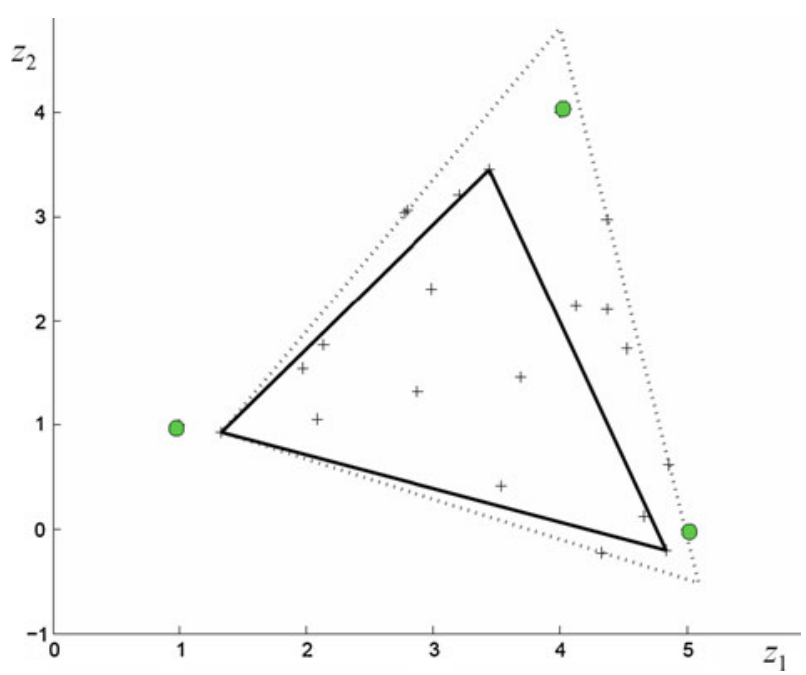

Fig. 5 Maximum volume $\tilde{S}$ (solid line) and minimum volume $\hat{S}$ (dotted line). Dots are endmember values in $V$ and crosses are 20 noisy observations $z_{k}$

Local nonlinear optimization may generate good approximations to the true endmembers, that usually correspond to correct estimates if noise is absent. This will be numerically illustrated in Sect. 4.

The exact number of points on the boundary of $S$ to recover $V$ by (2) depends on the number of zeros in the corresponding abundance vector $a$. In the extreme case, all pure constituents are present in the data. This means that in the data we have $n+1$ unit vectors $a_{k}=e_{i}$ being columns of the unit (identity) matrix $E$. The convex hull of the corresponding endmembers $v_{i}=V e_{i}$ encloses all observations in the absence of noise. Moreover, the volume of $S=\operatorname{conv}(V)$ is the largest if one selects $n+1$ points out of the $r$ observations, because they are convex combinations of $v_{i}$. The related largest volume problem is called the maximum volume simplex problem.

Consider the points in $Z$ now as a set $\mathbb{X}$. One wants to find that subset $\mathbb{V},|\mathbb{V}|=(n+1)$, with a corresponding simplex of maximum volume. Using notation of sets and matrices, one can express this as

$$
\max _{\mathbb{V} \subset \mathbb{X}}\left\{f(V):=\left|\operatorname{det}\left(\begin{array}{c}
V \\
\mathbf{1}^{T}
\end{array}\right)\right|\right\},
$$

where $V$ is a matrix with the columns of $\mathbb{V}$. Problem (4) is a combinatorial optimization problem. Let us call the solution of problem (4) $\tilde{V}$ and the corresponding simplex $\tilde{S}$. Notice that due to noise or absence of the endmembers in the data set, $\tilde{S}$ does not necessarily enclose all data points $z_{k}$. We illustrate this with an example.

Example 4 Consider the setting of Example 3 where now 20 points are generated with noise of $\sigma=0.3$. Figure 5 gives a minimum volume simplex $\hat{S}$ enclosing the points with volume 18.5. The maximum volume simplex $\tilde{S}$ that selects 3 points out of 20 has volume 11.25. It does not enclose all observations.

As illustrated by Example 4, the simplex $\hat{S}$ encloses all points, so also $\tilde{S} \subset \hat{S}$. However, the simplex $S$ that we intend to estimate does not have a fixed relation with them. It may be clear 
that lower noise and more observations as random combinations of the endmembers make $\tilde{S}$ go to $S$. Given fixed noise variance $\sigma^{2}$ and increasing a number of uniformly spread points, leads in limit to an overestimate of $S$, as observations fall outside $S$.

Problem (4) implies selecting $n+1$ points out of $r$. The number of possibilities grows as an $(n+1)$-degree polynomial in $r$. Usually, heuristic methods are used to find a good solution. The use of problem (4) for the estimation of parameters of model (1) is based on the assumption that endmembers are present in the data $Z$. N- FINDR [10] is a heuristic algorithm that generates a (not necessarily optimal) solution of problem (4) in the context of spectral unmixing applications. We refer here to N- FINDR as an algorithm and not to the commercial software that has been constructed around it. The N- FINDR algorithm is a so-called local search heuristic in the context of the available literature on combinatorial optimization, e.g. [1]. The algorithm has been extensively described in [10] with a claim that, under certain circumstances, it converges to the optimum solution of problem (4). We will use a MATLAB implementation of the algorithm as a reference method.

In the use of (2), noise complicates the estimation of $V$. More noise makes the volume of $\hat{S}$ bigger. Like in regression, one would like to draw the bounding facets through the exterior of the cloud of points instead of enclosing it. In [5] a procedure called MINVEST is developed based on that observation and compared to a multitude of methods for spectral unmixing. In Sect. 3, we describe the underlying ideas of this minimum volume based method. The next question is how sensitive such a minimum volume based method is to noise compared to a maximum volume based method. Does it still give accurate estimates if noise increases and local nonlinear search is used? We deal with that question in Sect. 4.

\section{A minimum volume simplex estimation procedure}

A procedure called MINVEST based on MVSEP is described to recover matrix $X$ and fraction vector $a_{k}$ from observations $y_{k}, k=1, \ldots, r$ of model (1). Despite data $y_{k} \in \mathbb{R}^{m}$, the corresponding ground truth data $X a_{k}$ lay in a lower dimensional space $\mathbb{Y}$, i.e. a point $y=X a \in \mathbb{Y}$ can be represented by a point $z \in \mathbb{R}^{n}$. Y can be described using an $m$-vector $b$ and an $m \times n$ orthonormal matrix $\Gamma$ and the relation:

$$
y=X a=b+\Gamma V a=b+\Gamma z
$$

where $z=V a$ is a $n$-vector that corresponds to $y$. Without noise, one can describe space $\mathbb{Y}$ with $n+1$ affine independent observations $y_{1} \ldots, y_{n+1}: \mathbb{Y}=y_{n+1}+<\left[y_{1}-y_{n+1}, \ldots, y_{n}-\right.$ $\left.y_{n+1}\right]>$, and determine $\Gamma$ accordingly. In the practical situation of having noise, one can estimate $\mathbb{Y}$ and a corresponding representation via vector $b$ and matrix $\Gamma$ by using principal component analysis. In that case, the choice for $b$ and $\Gamma$ is given by $b=\bar{y}=\frac{1}{r} \sum_{k} y_{k}$ and $\Gamma=C=\left[c_{1}, \ldots, c_{n}\right]$ is an $m \times n$ matrix of principal components; in direction $c_{1}$ we have the biggest variation, in direction $c_{2}$ the second biggest, etc. In this terminology, the transformed observations $z_{k}=C^{T}\left(y_{k}-\bar{y}\right)$ are called scores.

For the relation of model (3) and (1) in terms of noise, (5) implies that $\xi$ has $n$ Gaussian i.i.d. components with the same variance $\sigma^{2}$ due to $\Gamma$ being orthonormal; $\xi=\left(\Gamma^{T} \Gamma\right)^{-1} \Gamma^{T} \varepsilon=$ $\Gamma^{T} \varepsilon$. The procedure is to transform the data $y_{k}$ to $z_{k}$ in the lower dimensional space, create an estimate $\hat{V}$ of $V$ and to transform that back via $\hat{X}=C \hat{V}+\bar{y} \mathbf{1}^{T}$. As a second step, the abundance $a_{k}$ is estimated from $\hat{V}$ and $z_{k}$. 


\subsection{Estimate $\hat{V}$ of endmember matrix $V$}

Despite $V a$ can be found in the convex hull $S=\operatorname{conv}(V)$, observation $z_{k}$ may be out due to the noise in model (3). The idea for estimating $V$ is to base the final estimate of endmembers on the $P$ observations that we expect to be interior with respect to $S$. The endmembers are estimated iteratively from the minimum volume problem (2) where, at each iteration, the points at the boundary of its convex hull (active points) are removed until $P$ observations are left over. We estimate $P$ from a crude approximation, which assumes that if $a_{i k}=0$, the probability is $50 \%$ that the observation is in $S$. Assuming independence, with $N$ abundance values equal to zero we take that probability as $\left(\frac{1}{2}\right)^{N}$. Given that there are $r_{N}$ observations with ground truth $a_{k}$ having $N$ zeros, this estimate gives $P=\sum_{N=0}^{n}\left(\frac{1}{2}\right)^{N} r_{N}$. The values for $r_{N}$ in an experimental setting are found in the ground truth data $a_{k}$, or can be estimated from other estimation methods.

As starting simplex, one can take a random matrix or, for instance, the result of the N- FINDR algorithm. A solution of MVSEP (2) is determined, and points at the boundary are detected via the active (binding) constraints. These points are removed and (2) is again solved, points removed etc., until $P$ points are left over to base the estimate on. We illustrate Algorithm 1 with an example.

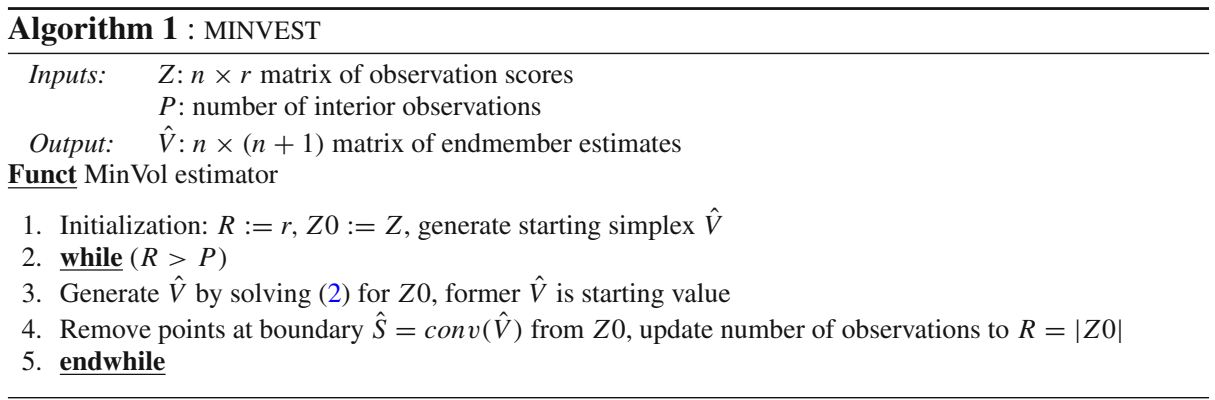

Example 5 Given the simplex of endmembers of Example 3, in total $r=100$ observations are generated on its boundary such that they have one zero abundance value each. Noise is added with $\sigma=0.2$. This means that about half of the observations are located inside; we can take $P=50$. The fmincon routine of MATLAB is used to solve (2) generating minimum volume enclosing simplices $\hat{S}$, given by dotted lines in Fig. 6 . In each iteration, about 6 points are active and removed from the set. $\hat{S}$ is determined again using the former estimate as starting value. This process continues up to the final estimate which in this case is based on 46 points. The estimated matrix of endmembers is given by $\hat{V}=\left(\begin{array}{ccc}1.02 & 5.03 & 4.00 \\ 1.03 & -0.02 & 4.26\end{array}\right)$ with a volume of 16.07 and corresponding simplex depicted by a solid line in Fig. 6. Notice that the fmincon solver generates local and not necessarily global solutions of (2). Despite the noise is considerable, one obtains adequate estimations of the simplex $S$.

\subsection{Estimate $\hat{A}$ of abundance matrix $A$}

To recover the fractional values $a_{k}$ from the estimated endmembers $V$, one can in principle solve 


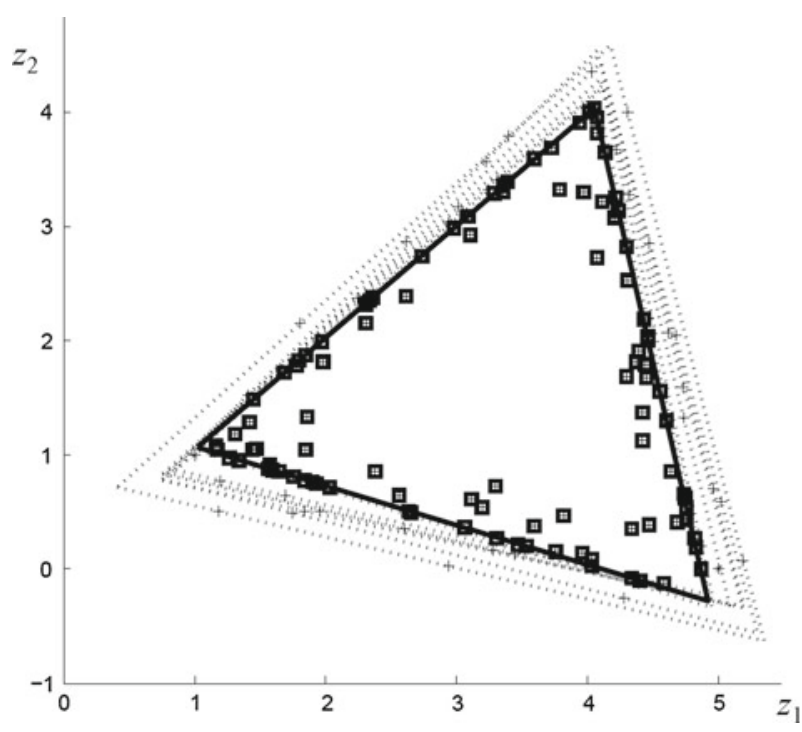

Fig. 6 Estimates of $S$, interior and projected observations represented as squares

$$
z_{k}=\left(\begin{array}{c}
V \\
\mathbf{1}^{T}
\end{array}\right)\left(\begin{array}{c}
a_{k} \\
1
\end{array}\right)
$$

as (6) implies having $(n+1)$ variables $a_{i k}$ and $(n+1)$ equations. For the fraction of observations located in the final set $Z 0$ and geometrically within simplex $\hat{S}$, we have automatically positive abundance values given by $\beta_{k}$ in (2). For observations $z_{k}$ outside $\hat{S}$, we have at least one corresponding $a_{j k}<0$. Assuming $\hat{S}=S$, the idea is that $z_{k}$ is located outside due to noise $\xi$. This makes it reasonable to project $z_{k}$ on the nearest facet of $\hat{S}$.

Let $\mathbb{F}$ be the space of that facet containing $p \leq n$ vertices called $u_{1}, \ldots, u_{p}$. Define matrix $W=\left[w_{1}, \ldots, w_{p-1}\right]$ with $w_{j}=u_{j}-u_{p}$. Now one can write

$$
\mathbb{F}=\left\{x=\sum_{j=1}^{p} a_{j} u_{j} \mid \sum_{j=1}^{p} a_{j}=1\right\}=u_{p}+\langle W\rangle .
$$

The relation between the two representations is that for an $(n+1)$-vector $a$ with $\sum_{j=1}^{p} a_{j}=$ $1 \rightarrow a_{p}=1-\sum_{j=1}^{p-1} a_{j}$, one can write point $x \in \mathbb{F}$ as

$$
x=\sum_{j=1}^{p} a_{j} u_{j}=u_{p}+\sum_{j=1}^{p-1} a_{j}\left(u_{j}-u_{p}\right)=u_{p}+\sum_{j=1}^{p-1} a_{j} w_{j} .
$$

The projection $x$ of $z$ on $\mathbb{F}$ is $x=W\left(W^{T} W\right)^{-1} W^{T}\left(z-u_{p}\right)$ and corresponding abundance

$$
a=\left(W^{T} W\right)^{-1} W^{T}\left(z-u_{p}\right), a \in \mathbb{R}^{p-1} \text { and } a_{p}=1-\sum_{j=1}^{p-1} a_{j} .
$$

The choice to make is which facet to project on, i.e. which $a_{j}$ are zero, so their $v_{j}$ should not be considered in matrix $U$. Let $\mathbb{J}(a)=\left\{j \mid a_{j}>0\right\}$ be the index set of positive values in a vector $a$. Algorithm 2 first determines $a$ via (6), selects endmembers $v_{j}$ from $\mathbb{J}(a)$ into 
matrix $U$, determines $W$ and calculates (9). Although not often, it may occur that the result still contains negative components. Those are set to zero and (9) is recalculated for the lower dimensional facet until only positive abundance values are left. In Fig. 6, the interior points $z$

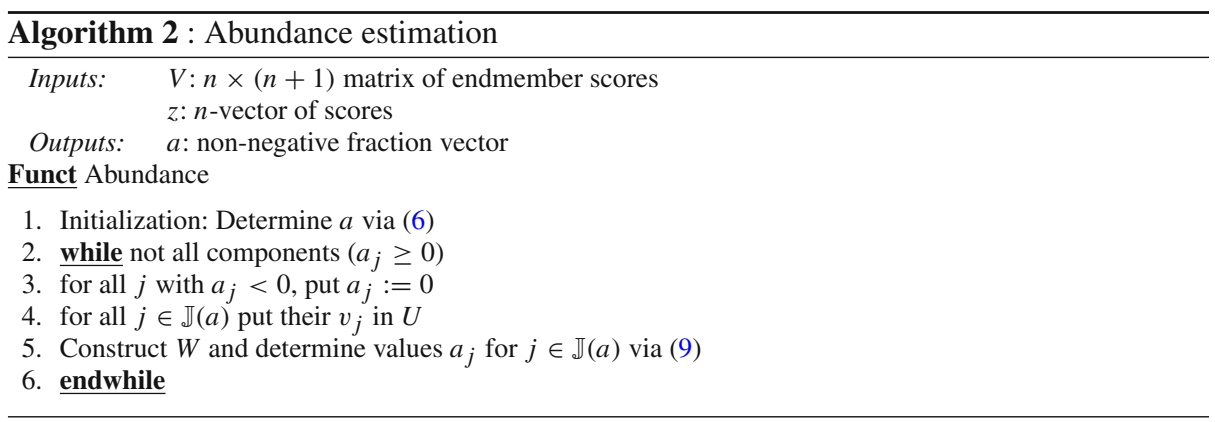

and the projected versions $x$ of the outside observations of Example 5 are given by squares. They correspond to nonnegative fractional values.

\section{Sensitivity with respect to noise}

As we have seen, without noise dimension reduction and enclosure lead to an exact recovery of the original parameters of the model. What is the influence of $\sigma$ on the accuracy of an MVSEP based method like MINVEST compared to a maximum volume simplex based method like N- FINDR?

To find an answer, we develop several experiments using simulations. The first experiment is a $4 \mathrm{D}$ instance where $\sigma$ is varied and the variance of the estimated values is measured. The second case is based on computer simulated hyperspectral scenes constructed from real spectral observations. It requires dimension reduction and further allows visual inspection of the results.

As a measure of viability of the estimation $\hat{V}$ of $\mathrm{V}$, we take the standard deviation assuming $\hat{V}$ is unbiased. This is also called root mean squared error (RMSE):

$$
\left.\sigma_{V}=\sqrt{(} \frac{1}{n(n+1)} \sum_{i} \sum_{j}\left(\hat{V}_{i j}-V_{i j}\right)^{2}\right) .
$$

The quality of estimation $\hat{A}$ of $A$ is measured as the standard deviation estimate assuming $\hat{A}$ is unbiased:

$$
\left.\sigma_{A}=\sqrt{(} \frac{1}{r \times n} \sum_{i} \sum_{k}\left(\hat{A}_{i k}-A_{i k}\right)^{2}\right) .
$$

We measure as well the variance $\sigma_{A}$ of $A$ generated by (6) (this is called linear spectral unmixing in the literature) as variance $\sigma_{A p}$ of $A$ generated by Algorithm 2. The latter is called fully constrained linear spectral unmixing (FCLSU) in the spectral unmixing literature.

\subsection{A 4D experiment}

This experiment has the following ingredients: 
Table 1 Standard deviation estimates (RMSE) of endmembers $V$ and abundance $A$ of by N- FINDR and MINVEST algorithms given noise standard deviation $\sigma$

\begin{tabular}{|c|c|c|c|c|c|c|c|c|c|c|}
\hline \multirow[t]{2}{*}{$\sigma$} & \multicolumn{5}{|c|}{ N- FINDR } & \multicolumn{5}{|c|}{ MINVEST } \\
\hline & 0.01 & 0.1 & 0.2 & 0.5 & 0.7 & 0.01 & 0.1 & 0.2 & 0.5 & 0.7 \\
\hline$\sigma_{V}$ & 0.030 & 0.118 & 0.233 & 0.857 & 1.359 & 0.013 & 0.111 & 0.194 & 0.486 & 0.922 \\
\hline$\sigma_{A}$ & 0.011 & 0.063 & 0.114 & 0.259 & 0.323 & 0.007 & 0.058 & 0.105 & 0.204 & 0.266 \\
\hline$\sigma_{A p}$ & 0.008 & 0.048 & 0.092 & 0.224 & 0.281 & 0.005 & 0.048 & 0.086 & 0.174 & 0.234 \\
\hline
\end{tabular}

- There are $(n+1)=5$ endmembers and $r=500$ observations in $\mathbb{R}^{4}$

- The endmember matrix to be estimated is $V=\left(\begin{array}{lllll}0 & 1 & 2 & 3 & 5 \\ 5 & 1 & 3 & 5 & 4 \\ 0 & 1 & 1 & 2 & 0 \\ 0 & 0 & 2 & 1 & 0\end{array}\right)$. We keep the order of endmembers fixed by sorting the first row. In this way, estimates $\hat{V}$ and $V$ are easily compared.

- To mimic the idea of combinations of a few constituents, a ground-truth abundance matrix $A$ is generated consisting of $50 \%$ of mixtures of 2 endmembers and $50 \%$ of mixtures of 3 endmembers. They are generated uniformly over the corresponding facets of the unit simplex.

- The score matrix $Z$ used as input for the estimation is taken as $Z=V A+\sigma \times \xi$, where $\xi$ is standard Gaussian noise. Given that the measured RMSE depends on (pseudo-)randomly drawn Gaussian noise, we replicate noise 100 times, i.e. generate replications for $Z$, and take the average of the measures.

- The estimate of the number of points $P$ that fall in the simplex is based on: $r_{0}=r_{1}=$ $r_{4}=0$ and $r_{2}=r_{3}=250$, so $P=93.75$.

The result for N- FINDR and MINVEST is given in Table 1. It shows standard deviation estimates $\sigma_{V}$ of endmembers and $\sigma_{A}$ of fractional abundances calculated via (6) and via FCLSU. One can observe that the standard deviation of the estimates is in the same order of magnitude as that of noise. This means that the procedures give results as accurate as the input data. Deviation of endmembers and abundances estimations provided by N- FINDR are larger than those obtained with MINVEST. The reason may be that the pure endmembers $v_{i}$ are not present in the data.

\subsection{Experiment with spectral data}

In this experiment, data are taken from the field of hyperspectral imaging sensors. More specifically, the reflectance spectra of $n+1=5$ US Geological Survey (USGS) mineral spectra (alunite, buddingtonite, calcite, kaolinite and muscovite, all signatures are available online $^{1}$ ) were used to build an endmember matrix $X$ with $m=224$. A square synthetic image scene of size $100 \times 100$ pixels is simulated. Mixtures $X a_{k}$ between the five endmembers have been generated to construct ground-truth fractional maps, such that the endmembers are located at the four corners and at the center of the image, and signature abundance $a$ decreases linearly away from the pure pixels. Gaussian noise with a signal-to-noise ratio or SNR ranging from 30:1 to 70:1 was added to the scene in order to simulate contributions

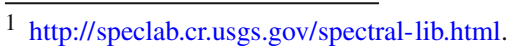


(a)

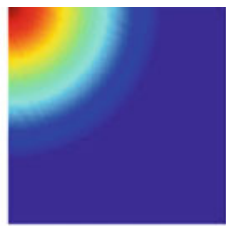

(b)

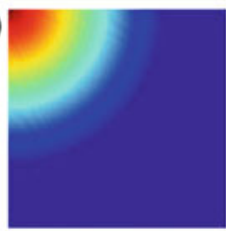

(c)

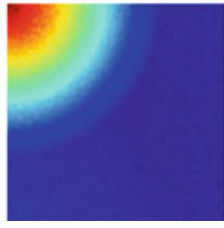

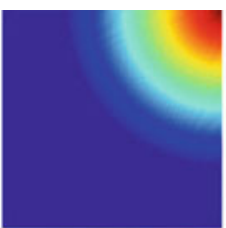
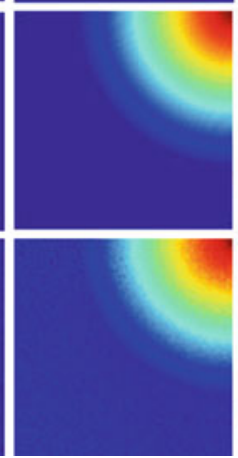
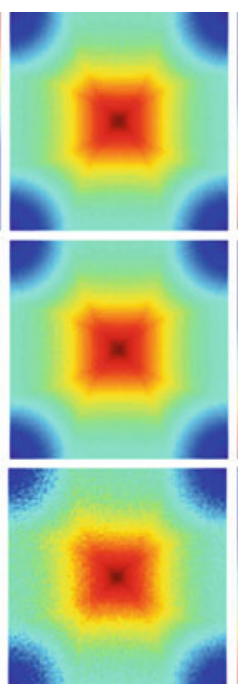
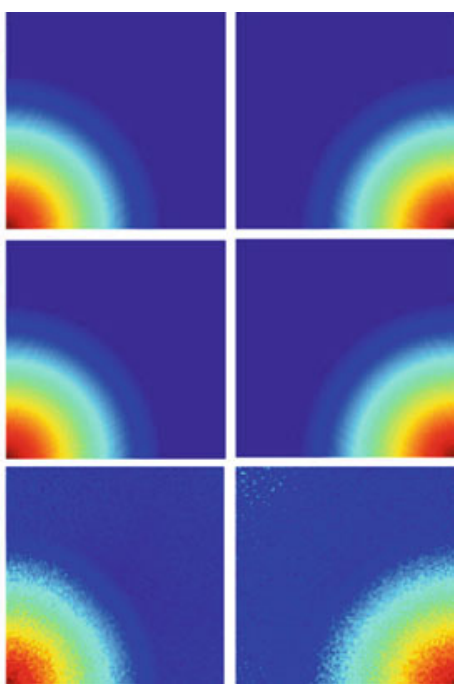

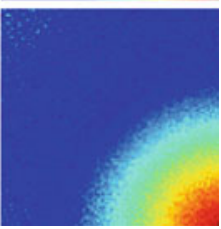

Fig. 7 a Ground-truth fractional abundance maps. b Fractional abundance estimations without noise using MINVEST algorithm. c Fractional abundance estimations for the image with SNR of 30:1 using MINVEST algorithm

from ambient (clutter) and instrumental sources. The signal-to-noise ratio is a usual way to express the ratio between the size of the observed property and the variance of the noise. Values higher than 70 are basically considered no-noise.

In hyperspectral analysis, the use of so-called fractional abundance maps is common to depict the fractional values for each endmember spatially. Figure 7a shows the ground-truth maps compared with the maps obtained by MINVEST for the simulated image without noise in Fig. 7b and with an SNR of 30:1 in Fig. 7c. The visual appearance of the fractional abundance maps generated by MINVEST algorithm is in very good compromise with the ground-truth fractional abundance maps, even for a scenario with high noise, i.e. the estimated abundance is always highly correlated to the abundances in the fractional maps of ground-truth values.

Figure 8 displays the fractional abundance estimations obtained by N- FINDR combined with a fully constrained least squares (FCLSU) algorithm for abundance estimation. It can be observed that the use of N- FINDR endmembers resulted in fractional maps which are not as properly distributed (in spatial terms) as those provided by MINVEST. In particular, this is the case for the simulated scene with SNR of 30:1 in Fig. 8c. Although the estimations resulting from N- FINDR are less correlated (i.e., they appear less similar visually) the RMSE of the estimated abundance fractions tells us if there are any scaling issues involved as was the case for some of the MINVEST estimations.

Table 2 gives the RMSE between the fractional abundance maps estimated for the endmembers extracted using N- FINDR (combined with FCLSU) and MINVEST. As shown by Table 2, the best estimations for the simulated scene without noise are provided by MINVEST, while the best estimations for the scenes with simulated noise are provided by N- FINDR. This seems not to be consistent with the visual appearance of the fractional abundance maps estimated by the different methods, in particular for the maps displayed in Fig. 8c and their visual agreement with the ground-truth maps. However, this is merely a scaling issue. Due to the dependence of MINVEST on the estimated number of observations falling inside the original simplex, the estimate of $S$ may over or underestimate its volume. This means that all abundance values are either too big or too small resulting in a higher RMSE value. The visual 

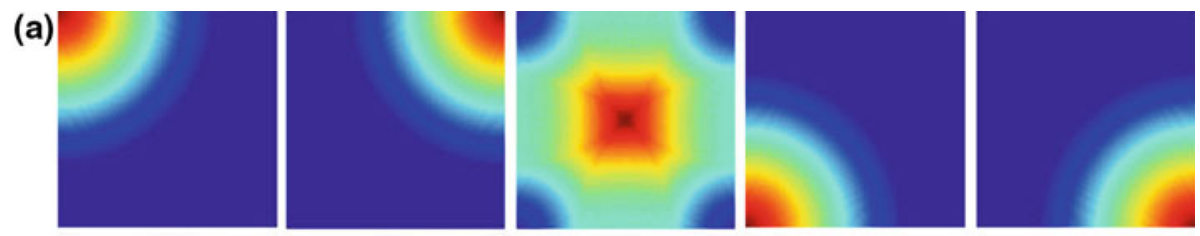

(b)
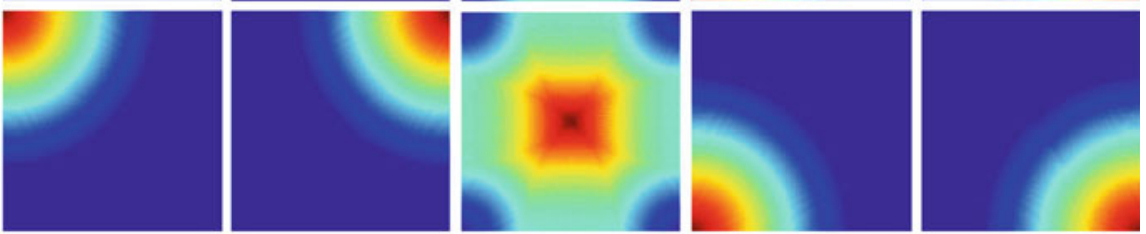

(c)
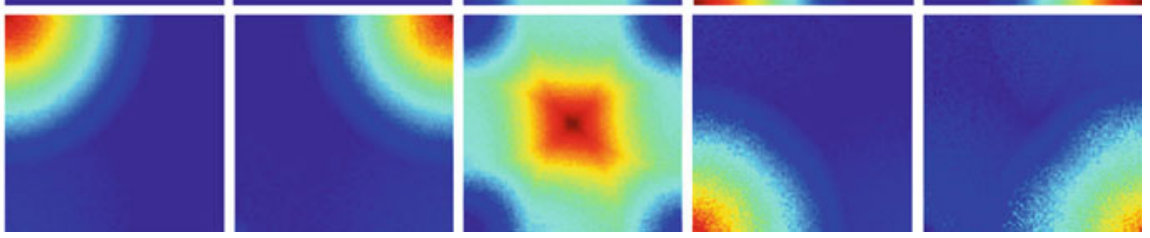

Fig. 8 a Ground-truth fractional abundance maps. b Fractional abundance estimations for the simulated image without noise using N- FINDR algorithm followed by fully constrained linear spectral unmixing. c Fractional abundance estimations for the simulated image with SNR of 30:1 using N- FINDR algorithm followed by fully constrained linear spectral unmixing

Table 2 RMSE values comparing fractional abundance estimated by different methods with true abundance; simulated scenes with different values for SNR

\begin{tabular}{lll}
\hline SNR & MINVEST & N- FINDR \\
\hline$\infty$ & 0.0000 & 0.0020 \\
70 & 0.0262 & 0.0067 \\
50 & 0.0347 & 0.0117 \\
30 & 0.0522 & 0.0331 \\
\hline
\end{tabular}

correspondence (correlation) tells us that apparently the orientation of the generated simplex by MINVEST is better than that generated by N- FINDR. A further study [5] goes into the scaling issue and compares the MINVEST algorithm described here with 10 other algorithms for spectral unmixing on benchmark cases.

\section{Conclusions}

The minimum volume simplicial enclosure problem (MVSEP) has been analyzed with a focus on its use for estimating endmember and abundance parameters in a linear mixing model. An estimation method called MINVEST has been described and its behaviour for the variance in the input data has been investigated and compared to a maximum volume simplex based method called N- FINDR.

The MVSEP is a global optimization problem where the number of optima depends in the worst case on the number of points in the convex hull of the instance. The result of the combinatorial optimization problem of finding a maximum volume simplex by selecting data as vertices is logically enclosed by the result of the MVSEP. Both problems are used as a basis for estimating the parameters in a linear mixing model. 
For data from linear mixing models that are spread over the boundary of the simplex, the MVSEP has one minimum that, due to permutations, has a number of minimum points. Surprisingly, local search leads in general to the global optimum enclosing simplex of MVESP. Moreover, if data are generated from the mixing model without any noise, the MVSEP based MINVEST method returns the original endmember values. This means that no pure pixels (endmembers) are required to be present in the data set. Maximum volume based methods like N- FINDR require the assumption of presence of pure points.

The variation in the estimation results reflects the variance in the input data. Even when using nonlinear local search in MINVEST, the variation is of the same order of magnitude whereas the N- FINDR method seems more sensitive to high variance. The results of MINVEST seem more correlated to ground-truth abundance data than the ones of N- FINDR. However, the values for the root mean squared error performance indicator appear sensitive to scaling in its use for measuring abundance discrepancies.

Further research can focus on the relation between the used values for the estimated number $P$ of interior points and the scaling effects observed in the abundance discrepancy measure.

Acknowledgments The authors gratefully thank the Guest Editors and the Anonymous Reviewers for their outstanding comments and suggestions, which greatly helped us to improve the technical quality and presentation of the manuscript.

Open Access This article is distributed under the terms of the Creative Commons Attribution License which permits any use, distribution, and reproduction in any medium, provided the original author(s) and the source are credited.

\section{References}

1. Aarts, E.H.L., Lenstra, J.K.: Local Search Algorithms. Wiley, New York (1997)

2. Bioucas-Dias, J.M., Plaza, A.: Hyperspectral unmixing: geometrical, statistical and parse regression-based approaches. SPIE Remote Sensing Europe Conference (2010)

3. Craig, M.D.: Minimum-volume transforms for remotely sensed data. IEEE Trans. Geosci. Remote Sens. 32(3), 542-552 (1994)

4. Hendrix, E.M.T., Toth, B.G.: Introduction to Nonlinear and Global Optimization. Springer, New York (2010)

5. Hendrix, E.M.T., García, I., Plaza, J., Martín, G., Plaza, A.: A new minimum volume enclosing algorithm for endmember identification and abundance estimation in hyperspectral data. IEEE Trans. Geosci. Remote Sens (2012). doi:10.1109/TGRS.2011.2174443

6. Keshava, N.: A survey of spectral unmixing algorithms. Lincoln Lab. J. 14, 55-78 (2003)

7. Khachiyan, L.G., Todd, M.J.: On the complexity of approximating the maximal inscribed ellipsoid for a polytope. Math. Program. 61, 137-159 (1993)

8. Meijer, E., Buurman, P.: Factor analysis and direct optimization of the amounts and properties of volcanic soil constituents. Geoderma 80, 129-151 (1997)

9. Sun, P., Freund, R.M.: Computation of minimum-volume covering ellipsoids. Oper. Res. 52(5), 690706 (2004)

10. Winter, M.E.: N-FINDR: an algorithm for fast autonomous spectral end-member determination in hyperspectral data. Proc. SPIE Image Spectrom. V 3753, 266-277 (2003)

11. Zhou, Y., Suri, S.: Algorithms for a minimum volume enclosing simplex in three dimensions. SIAM J. Comput. 31(5), 1339-1357 (2002) 